\title{
Public revenue in India: Trend and effect
}

\author{
Devasia M D ${ }^{1}$, N Karunakaran ${ }^{2 *}$ \\ ${ }^{\mathbf{1}}$ Associate Professor and Research Guide, ${ }^{2}$ Principal and Research Guide, ${ }^{1}$ Dept. of Economics, Nirmalagiri College, Kuthuparamba, \\ Kannur, Kerala, India, ${ }^{2}$ Economics of Kanpur University, People Institute of Management Studies (PIMS), Kasaragod, Kerala, India
}

\section{*Corresponding Author: N Karunakaran}

Email: narankarun@gmail.com

\begin{abstract}
Public revenue is a major component of budget shows the manner in which revenue is collected during a financial year by government to boost economic growth. The success of government plan for the growth and development of a country depends on the source and size of public revenue. In India Public revenue acts as government's most important economic and fiscal policy tool in controlling money supply and maintaining general price level. It is not only important for the corporate but for individuals from all sections of the society as they look forward to tax exemptions and reliefs. Even though India ranks third in purchasing power parity, only a few percentages of population pay income tax. The government's effort to widen the tax base has resulted in an 80 percent jump in number of IT returns filed. The analysis revealed that Public Revenue and macroeconomic indicators are significantly interlinked and correlated. Tax buoyancy is an indicator to measure efficiency to growth in GDP and Gross tax buoyancy coefficient remained fluctuating during the period 1990-91 to 2016-17.
\end{abstract}

Keywords: Budget, Revenue, Capital, Tax, GDP, Trend, Effect.

\section{Introduction}

Government budget is framed in the shape of a financial plan which is a statement of income and expenditure relating to various economic and other activities that the government intends to perform in the coming period, usually a year. Budget not only shows the public receipt and expenditure but also the attitude of government towards its people, what value it given to the people and priorities given by the country for different sectors of the economy (Sonika Gupta K. S, 2016). The structure of budget frame may be different in different countries. In India, government account of the budget are presented in three parts, viz.,(a) Consolidated fund, (b) Contingency fund and (c) Public account fund. Nearly, 20 percent of public receipt comes from borrowings and other liabilities, and the corporate tax is the major source of revenue after implementing GST. Though, India is third rank in purchasing power parity (Sidhartha, 2020), only a few percentage of people pay income tax. In the last four years, the government's efforts to widen the tax base resulted in an 80 percent jump in number of IT returns filed to 6.85 crore in 2017-18 from 3.31crore in 2013-14. 2.02 crore individuals filed incometax returns in 2017-18 declaring their income, but paid zero tax since they are not in the taxable income bracket yet; the number of such companies was 3.9 lakh. So it is very essential to analyze the trend, pattern and effects of public revenue in India; more specifically, how India obtains its revenue resource for making development and the impact of such revenue on real macro variables.

\section{Materials and Methods}

The specific objectives of the paper are: (a) to analyze the trend, pattern and composition of revenue of the central government of India; and (b) to examine the impact of public revenue on major macroeconomic variables. The study is based on the secondary data collected from various volumes of RBI Bulletin, Central Budget Document in different years mainly 2000 to 2019, Economic Survey, Central Statistical Office Publications, Indian Public Finance Statistical Year Book, Report of Planning Commission and Census of India. For analytical purpose, the period of study (1991-2019) is divided into three: 19902000, 2000-10 and 2010-19. For analysis correlation and regression were used.

\section{Results, Analysis and Discussion Trend and pattern of public revenue}

Government raises its fund to finance their activities from various sources like tax and non-tax sources, currency mint, fees, fines, sale of public assets, and so on (Rajan Goyal, J. $\mathrm{K}$, 2004). Adam Smith divided public revenue as revenue from the people and revenue from state property; while Dalton preferred to distinguish between public receipts and public revenue (Seligman, 1892).

\section{Classification of receipts}

Budget 1957-58 divided the income of central government into revenue and capital account. Revenue receipts include revenue received in the form of tax and non-tax revenue and capital account composed of market borrowing, small saving, provident fund, special deposit, recovery of loans, disinvestment receipts, and external loan (Devasia M D, Karunakaran N and Vishnu Prathap M, 2020).

Table 1 shows the distribution of revenue and capital receipts. Total receipts continuously increasing since 1990, from Rs. 93951 crore in $1990-91$ to 2315113 crore in 201819, showing nearly four-fold increase in total receipt (Fig. 1). In the first phase of $1990-99,60.8$ percent of total receipt is from revenue receipts; in the second (62 percent) and third phase ( 65 percent). The major reason behind this is due to the significant measures taken by government of India for 
augmenting revenue such as reduced tax rate and increased coverage of tax system (Odedokun, 2001; Sonika, 2016).

Table 1: Total receipts of the Central Government of India

\begin{tabular}{|c|c|c|c|c|c|c|}
\hline \multirow[b]{2}{*}{ Year } & \multicolumn{2}{|c|}{ Revenue Receipts } & \multicolumn{2}{|c|}{ Capital Receipts } & \multicolumn{2}{|c|}{ Total Receipts } \\
\hline & (percent) & (Rs. Crore) & (percent) & (Rs. Crore) & (percent) & (Rs. Crore) \\
\hline 1990-91 & 58.49 & 54954 & 41.51 & 38997 & 100 & 93951 \\
\hline $1991-92$ & 63.15 & 66030 & 36.85 & 38528 & 100 & 104558 \\
\hline $1992-93$ & 67.20 & 74128 & 32.80 & 36178 & 100 & 110306 \\
\hline 1993-94 & 57.64 & 75453 & 42.36 & 55440 & 100 & 130893 \\
\hline $1994-95$ & 57.01 & 91083 & 42.99 & 68695 & 100 & 159778 \\
\hline $1995-96$ & 65.36 & 110103 & 34.63 & 58338 & 100 & 168468 \\
\hline $1996-97$ & 67.23 & 126279 & 32.77 & 61544 & 100 & 187823 \\
\hline $1997-98$ & 57.47 & 133886 & 42.53 & 99077 & 100 & 232963 \\
\hline $1998-99$ & 53.47 & 149485 & 46.53 & 130064 & 100 & 279549 \\
\hline 1999-00 & 61.07 & 181482 & 38.93 & 115707 & 100 & 297189 \\
\hline Average & 60.81 & 106288.3 & 39.19 & 70256.8 & 100 & 176547.8 \\
\hline $2000-01$ & 58.94 & 192605 & 41.06 & 134184 & 100 & 326789 \\
\hline $2001-02$ & 55.33 & 201306 & 44.67 & 162500 & 100 & 363806 \\
\hline $2002-03$ & 56.11 & 230834 & 43.89 & 180531 & 100 & 411365 \\
\hline 2003-04 & 55.52 & 263813 & 44.48 & 211333 & 100 & 475146 \\
\hline 2004-05 & 60.43 & 305991 & 39.57 & 200391 & 100 & 506382 \\
\hline 2005-06 & 65.91 & 347077 & 34.09 & 179549 & 100 & 526626 \\
\hline $2006-07$ & 75.04 & 434387 & 24.96 & 144482 & 100 & 578869 \\
\hline $2007-08$ & 73.24 & 541864 & 26.76 & 197978 & 100 & 739842 \\
\hline $2008-09$ & 64.31 & 540259 & 35.69 & 299863 & 100 & 840122 \\
\hline $2009-10$ & 55.84 & 572811 & 44.16 & 453063 & 100 & 1025874 \\
\hline Average & 62.07 & 363094.7 & 37.93 & 216387.4 & 100 & 579482.1 \\
\hline $2010-11$ & 66.21 & 788471 & 33.79 & 402428 & 100 & 1190899 \\
\hline $2011-12$ & 56.91 & 751437 & 43.09 & 568918 & 100 & 1320355 \\
\hline $2012-13$ & 59.35 & 879232 & 39.30 & 582152 & 100 & 1481383 \\
\hline 2013-14 & 64.28 & 1014724 & 35.72 & 563894 & 100 & 1578618 \\
\hline $2014-15$ & 69.45 & 1101381 & 30.55 & 484448 & 100 & 1585829 \\
\hline 2015-16 & 66.73 & 1195025 & 33.27 & 595748 & 100 & 1790783 \\
\hline 2016-17 & 69.57 & 1374203 & 30.43 & 600991 & 100 & 1975194 \\
\hline $2017-18$ & 67.01 & 1435233 & 32.99 & 706740 & 100 & 2141973 \\
\hline $2018-19$ & 67.08 & 1552916 & 32.92 & 762197 & 100 & 2315113 \\
\hline Average & 65.18 & 1121402 & 34.67 & 585279.6 & 100 & 1708905 \\
\hline
\end{tabular}

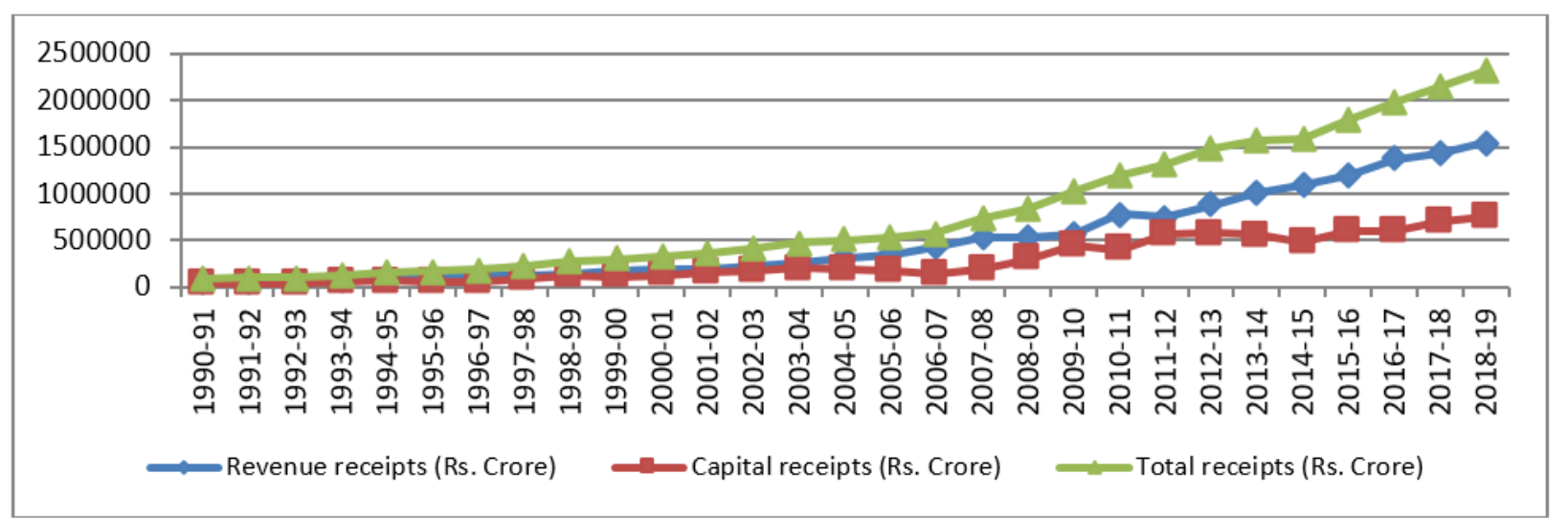

Fig. 1: Trend of Governments Receipts (1990-2019) 


\section{Components of revenue receipt}

Total revenue receipts are classified into two heads; Tax revenue and Non-tax revenue. The tax revenue includes income tax, corporation tax, excise duty and custom duty. The principal sources of non-tax revenue are interest receipts, net contribution of public sector undertaking, fiscal services, general services, social and community services, economic services and external grants (Brown J. L and Howard L. R, 2002).

Table 2: Share of tax and non-tax revenue in total revenue receipts

\begin{tabular}{|c|c|c|c|c|c|c|}
\hline \multirow[b]{2}{*}{ Year } & \multicolumn{2}{|c|}{ Tax Revenue } & \multicolumn{2}{|c|}{ Non tax Revenue } & \multicolumn{2}{|c|}{ Total Revenue Receipts } \\
\hline & (percent) & (Rs. crore) & (percent) & (Rs .crore) & (percent) & (Rs. crore) \\
\hline $1990-91$ & 78.21 & 42978 & 21.79 & 11976 & 100 & 54954 \\
\hline $1991-92$ & 75.83 & 50069 & 24.17 & 15961 & 100 & 66030 \\
\hline $1992-93$ & 72.91 & 54044 & 27.09 & 20084 & 100 & 74128 \\
\hline $1993-94$ & 70.84 & 53449 & 29.16 & 22004 & 100 & 75453 \\
\hline 1994-95 & 74.06 & 67454 & 25.94 & 23629 & 100 & 91083 \\
\hline $1995-96$ & 74.42 & 81939 & 25.60 & 28191 & 100 & 110103 \\
\hline $1996-97$ & 74.20 & 93701 & 25.80 & 32578 & 100 & 126279 \\
\hline $1997-98$ & 71.46 & 95672 & 28.54 & 38214 & 100 & 133886 \\
\hline $1998-99$ & 70.01 & 104652 & 29.99 & 44833 & 100 & 149485 \\
\hline 1999-00 & 70.68 & 128271 & 29.32 & 53211 & 100 & 181482 \\
\hline Average & 73.26 & 77222.9 & 26.74 & 29068.1 & 100 & 106288.3 \\
\hline $2000-01$ & 70.95 & 136658 & 29.05 & 55947 & 100 & 192605 \\
\hline 2001-02 & 66.33 & 133532 & 33.67 & 67774 & 100 & 201306 \\
\hline 2002-03 & 68.68 & 158544 & 31.32 & 72290 & 100 & 230834 \\
\hline 2003-04 & 70.88 & 186982 & 29.12 & 76831 & 100 & 263813 \\
\hline 2004-05 & 73.47 & 224798 & 26.53 & 81193 & 100 & 305991 \\
\hline $2005-06$ & 77.87 & 270264 & 22.13 & 76813 & 100 & 347077 \\
\hline 2006-07 & 80.85 & 351182 & 19.15 & 83205 & 100 & 434387 \\
\hline $2007-08$ & 81.12 & 439547 & 18.88 & 102317 & 100 & 541864 \\
\hline 2008-09 & 82.06 & 443319 & 17.94 & 96940 & 100 & 540259 \\
\hline $2009-10$ & 79.70 & 456536 & 20.30 & 116275 & 100 & 572811 \\
\hline Average & 75.19 & 280136 & 24.81 & 82958.5 & 100 & 363094.7 \\
\hline $2010-11$ & 72.28 & 569868 & 27.72 & 218602 & 100 & 788471 \\
\hline 2011-12 & 83.81 & 629764 & 16.19 & 121672 & 100 & 751437 \\
\hline 2012-13 & 84.38 & 741877 & 15.62 & 137354 & 100 & 879232 \\
\hline 2013-14 & 80.40 & 815854 & 19.60 & 198870 & 100 & 1014724 \\
\hline 2014-15 & 82.04 & 903615 & 17.96 & 197766 & 100 & 1101381 \\
\hline $2015-16$ & 78.97 & 943765 & 21.03 & 251260 & 100 & 1195025 \\
\hline 2016-17 & 80.15 & 1101372 & 19.85 & 272831 & 100 & 1374203 \\
\hline 2017-18 & 86.57 & 1242488 & 13.43 & 192745 & 100 & 1435233 \\
\hline 2018-19 & 84.82 & 1317211 & 15.18 & 235705 & 100 & 1552916 \\
\hline Average & 81.49 & 918423.8 & 18.51 & 202978 & 100 & 1121402.4 \\
\hline
\end{tabular}

Table 2 shows a rapid increase in the share of tax revenue of central government from Rs. 42978 crore in 1990 to Rs. 918423 crore in 2018-19. During the first phase (1990-1999), 73 percent of it is contributed by tax revenue, though a slight decline in its percentage is visible, the trend has maintained in the second and third phase too (Fig. 2).

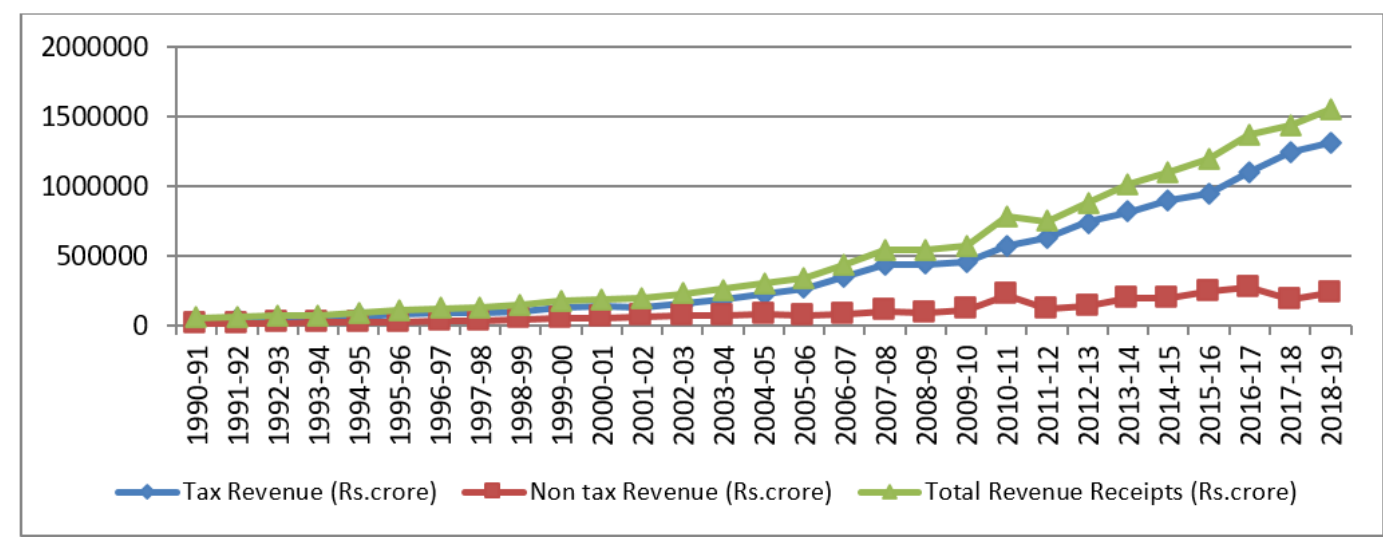

Fig. 2: Trend of govt's revenue receipts 1990-2019 


\section{Major component of capital receipts}

Those receipts of central government which create liability and reduce financial asset are called capital receipts (Barry Bosworth S. M, 2008). These include loans raised by the government from public which are called market loans, borrowing by the government from Reserve Bank of India and others through sale of Treasury Bills, loans received from foreign governments and bodies and recoveries of loans granted by the central government to state and union territory governments and others (Odedokun M. O, 2001). It also included proceeds from disinvestment of government equity in public enterprises. Capital receipt also show an increase from time to time; from Rs. 38997 crore in 1990-91 to Rs. 618120 crore (Table 3).

Table 3: Major component of Capital Receipts (As a percent of Total)

\begin{tabular}{|c|c|c|c|c|c|}
\hline Year & $\begin{array}{c}\text { Market Loans } \\
\text { (net) }\end{array}$ & $\begin{array}{l}\text { External } \\
\text { Debt(net) }\end{array}$ & Small Saving & Other Receipts & Capital Receipts (crore) \\
\hline $1990-91$ & 20.52 & 8.16 & 21.31 & 50.02 & 38997 \\
\hline $1991-92$ & 19.49 & 14.07 & 14.68 & 51.76 & 38528 \\
\hline $1992-93$ & 10.16 & 14.70 & 12.09 & 63.05 & 36178 \\
\hline 1993-94 & 52.18 & 9.15 & 12.91 & 25.76 & 55440 \\
\hline $1994-95$ & 29.59 & 5.21 & 21.03 & 44.17 & 68695 \\
\hline $1995-96$ & 58.28 & 0.55 & 17.32 & 23.85 & 58338 \\
\hline $1996-97$ & 31.02 & 4.85 & 19.78 & 44.34 & 61544 \\
\hline $1997-98$ & 32.80 & 1.10 & 20.65 & 45.44 & 99077 \\
\hline $1998-99$ & 53.04 & 1.48 & 25.40 & 20.08 & 130064 \\
\hline 1999-00 & 53.65 & 1.02 & 7.76 & 37.57 & 115707 \\
\hline Average & 36.1 & 6 & 17.3 & 40.6 & 70256.8 \\
\hline $2000-01$ & 54.72 & 5.59 & 6.20 & 33.49 & 13414 \\
\hline 2001-02 & 55.88 & 3.45 & 5.39 & 35.28 & 162500 \\
\hline $2002-03$ & 57.68 & -6.61 & 3.73 & 45.20 & 180531 \\
\hline 2003-04 & 42.05 & -6.38 & -2.67 & 67.00 & 211333 \\
\hline 2004-05 & 25.42 & 7.36 & 22.38 & 44.84 & 200391 \\
\hline $2005-06$ & 59.17 & 4.16 & -6.26 & 42.92 & 179549 \\
\hline $2006-07$ & 72.54 & 5.86 & -9.57 & 31.17 & 144482 \\
\hline 2007-08 & 65.97 & 4.71 & -5.71 & 35.04 & 197978 \\
\hline $2008-09$ & 82.36 & 3.67 & -0.43 & 14.40 & 299863 \\
\hline $2009-10$ & 87.05 & 2.44 & 2.93 & 7.59 & 453063 \\
\hline Average & 60.28 & 2.42 & 1.6 & 35.69 & 216387.4 \\
\hline $2010-11$ & 81.11 & 5.85 & 2.79 & 10.25 & 402428 \\
\hline $2011-12$ & 85.09 & 2.19 & -1.81 & 14.53 & 568918 \\
\hline 2012-13 & 87.17 & 1.24 & 1.48 & 10.11 & 582152 \\
\hline 2013-14 & 84.35 & 1.29 & 2.19 & 12.17 & 563894 \\
\hline 2014-15 & 94.46 & 2.67 & 6.65 & -3.78 & 484448 \\
\hline $2015-16$ & 77.50 & 2.19 & 9.01 & 11.31 & 582579 \\
\hline 2016-17 & 73.86 & 2.70 & 16.41 & 7.02 & 550617 \\
\hline 2017-18 & 56.34 & 2.55 & 16.20 & 24.91 & 618120 \\
\hline Average & 77.80 & 2.57 & 6.06 & 13.58 & 507727.04 \\
\hline
\end{tabular}

During the first phase its average share was 36 percent, increased sharply to 60.28 percent in second phase and to 77.8 percent in third phase; showing, market loans as one of the important revenue source of central government. In the case of small savings, an up-down movement, similarly, the share of other capital receipts like provident fund, special deposit, etc. has shown a decreasing at speedy rate (Fig. 3). 


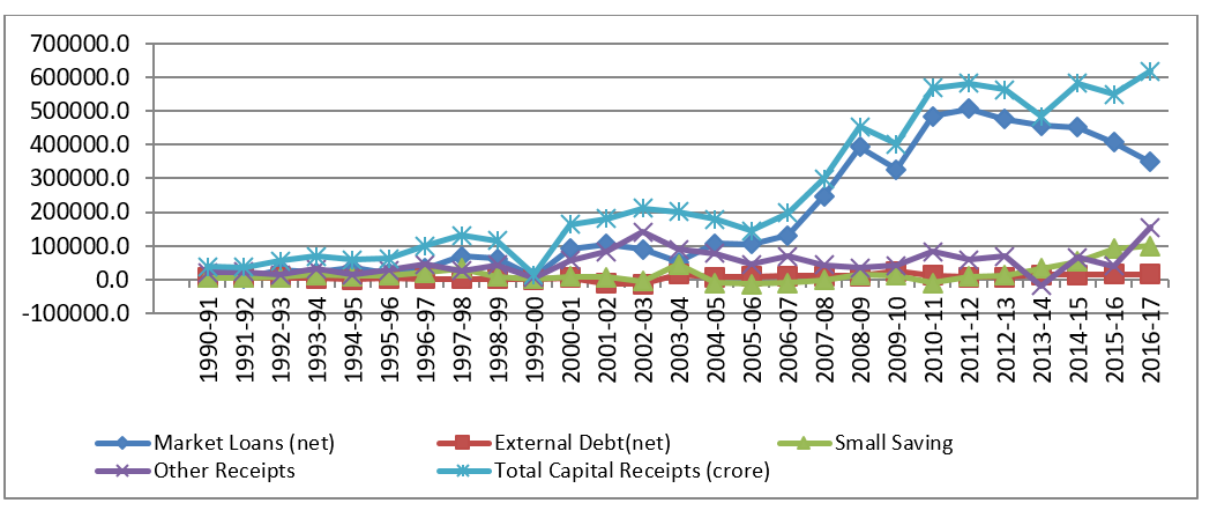

Fig. 3: Trend of governments capital receipts 1990 - 2019 (Rs. in Crore)

\section{Major components of direct taxes}

Direct tax is a type of tax where the incidence and impact of taxation fall on the same entity and tax burden can't be shifted by taxpayer to someone else. These are largely taxes on income or wealth, corporation tax, property tax, inheritance tax and gift tax. It is observed that there is a rapid increase in the share of direct taxes in total tax revenue. From 1990 to 2017 , the share of personal income tax in direct tax increased (Table 4); in the first phase it was 17.78 percent, increased to 37.2 percent and 38.24 percent in second and third phase, respectively.

Table 4: Major components of direct taxes

\begin{tabular}{|c|c|c|c|c|c|c|c|}
\hline \multirow[b]{2}{*}{ Year } & \multicolumn{2}{|c|}{ Personal Income Tax } & \multicolumn{2}{|c|}{ Corporation Tax } & \multicolumn{2}{|c|}{ Other Direct Taxes } & \multirow[b]{2}{*}{ Direct Tax (crore) } \\
\hline & $\%$ & Rs. Crore & Percent & Rs. Crore & Percent & Rs. Crore & \\
\hline $1990-91$ & 18.11 & 1250 & 77.29 & 5335 & 4.61 & 318 & 6903 \\
\hline $1991-92$ & 16.10 & 1627 & 77.73 & 7853 & 6.17 & 623 & 10103 \\
\hline $1993-94$ & 10.82 & 1355 & 80.34 & 10060 & 8.84 & 1107 & 12522 \\
\hline $1994-95$ & 18.84 & 3468 & 75.08 & 13822 & 6.08 & 1119 & 18409 \\
\hline $1997-98$ & 13.21 & 3589 & 73.66 & 20016 & 13.13 & 3567 & 27172 \\
\hline $1998-99$ & 17.93 & 5760 & 76.37 & 24529 & 5.70 & 1831 & 32120 \\
\hline 1999-00 & 22.04 & 9131 & 74.07 & 30692 & 3.89 & 1613 & 41436 \\
\hline Average & 17.78 & 3704.4 & 74.98 & 15626 & 7.24 & 1510.1 & 20840.1 \\
\hline $2000-01$ & 47.87 & 23766 & 50.71 & 25177 & 1.43 & 708 & 49651 \\
\hline 2004-05 & 36.94 & 35443 & 62.84 & 60289 & 0.22 & 212 & 95944 \\
\hline $2005-06$ & 37.48 & 45238 & 62.30 & 75187 & 0.22 & 267 & 120692 \\
\hline $2006-07$ & 36.94 & 62707 & 62.86 & 106701 & 0.19 & 330 & 169738 \\
\hline $2007-08$ & 37.38 & 86563 & 62.47 & 144660 & 0.15 & 351 & 231574 \\
\hline $2008-09$ & 35.05 & 86985 & 64.80 & 160797 & 0.15 & 370 & 248152 \\
\hline $2009-10$ & 34.80 & 94532 & 65.09 & 176797 & 0.11 & 294 & 271623 \\
\hline Average & 37.20 & 51088.4 & 62.21 & 85434 & .59 & 805.5 & 137327.9 \\
\hline $2010-11$ & 32.68 & 102441 & 66.70 & 209115 & 0.62 & 1945 & 313501 \\
\hline $2011-12$ & 34.44 & 118224 & 66.24 & 227411 & -0.68 & -2325 & 343310 \\
\hline $2012-13$ & 35.41 & 140438 & 64.44 & 255570 & 0.15 & 577 & 396585 \\
\hline $2013-14$ & 37.16 & 169408 & 62.69 & 285742 & 0.15 & 679 & 455829 \\
\hline
\end{tabular}


Trend of major components of indirect taxes

Indirect taxes are basically taxes/burden that can be shifted on to another entity or ultimate buyer, viz; sales tax (CGST/SGST), excise duty, custom duty, etc. Table 5 shows the share of Excise duty and Custom duty in total Indirect Tax. There is a rapid increase in the share of indirect taxes in total tax revenue from Rs. 36075 crore in 1990-91 to Rs. 620716 crore in 2017-18. Compared to direct taxes, the share of indirect tax has shown a speedy rise.

Table 5: Share of excise duty and custom duty in total indirect tax

\begin{tabular}{|l|c|c|c|c|c|c|}
\hline \multirow{2}{*}{ Year } & \multicolumn{2}{|c|}{ Excise duty } & \multicolumn{2}{c|}{ Custom duty } & \multicolumn{2}{c|}{ Indirect Tax } \\
\cline { 2 - 7 } & Percent & Rs. Crore & Percent & Rs. Crore & Percent & Rs. Crore \\
\hline $1990-91$ & 39.09 & 14100 & 57.23 & 20644 & 100 & 36075 \\
\hline $1991-92$ & 40.08 & 16017 & 55.69 & 22257 & 100 & 39966 \\
\hline $1992-93$ & 39.00 & 16367 & 56.65 & 23776 & 100 & 41969 \\
\hline $1993-94$ & 42.08 & 17224 & 54.23 & 22193 & 100 & 40927 \\
\hline $1994-95$ & 42.95 & 21064 & 54.62 & 26789 & 100 & 49045 \\
\hline $1995-96$ & 37.18 & 22176 & 59.94 & 35757 & 100 & 59652 \\
\hline $1996-97$ & 34.34 & 23463 & 62.72 & 42851 & 100 & 68326 \\
\hline $1997-98$ & 37.25 & 25516 & 58.68 & 40193 & 100 & 68500 \\
\hline $1998-99$ & 39.40 & 28581 & 56.07 & 40668 & 100 & 72532 \\
\hline $1999-00$ & 40.24 & 34944 & 55.76 & 48419 & 100 & 86836 \\
\hline Average & 39.16 & 21945.2 & 57.16 & 32354.7 & 100 & 56382.8 \\
\hline $2000-01$ & 57.19 & 49758 & 39.26 & 34163 & 100 & 87007 \\
\hline $2001-02$ & 63.46 & 54469 & 33.02 & 28340 & 100 & 85828 \\
\hline $2002-03$ & 64.36 & 62388 & 32.91 & 31898 & 100 & 96939 \\
\hline $2003-04$ & 63.63 & 70245 & 31.33 & 34586 & 100 & 110392 \\
\hline $2004-05$ & 59.94 & 77241 & 32.45 & 41811 & 100 & 128854 \\
\hline $2005-06$ & 57.93 & 86642 & 31.19 & 46645 & 100 & 149572 \\
\hline $2006-07$ & 51.06 & 92651 & 34.62 & 62819 & 100 & 181444 \\
\hline $2007-08$ & 46.25 & 96178 & 36.25 & 75382 & 100 & 207972 \\
\hline $2008-09$ & 41.95 & 81872 & 35.47 & 69217 & 100 & 195169 \\
\hline $2009-10$ & 45.63 & 84383 & 32.57 & 60223 & 100 & 184913 \\
\hline Average & 55.14 & 75582.7 & 33.91 & 48508.4 & 100 & 142809 \\
\hline $2010-11$ & 42.99 & 110222 & 36.12 & 92598 & 100 & 256367 \\
\hline $2011-12$ & 40.57 & 116226 & 36.87 & 105614 & 100 & 286454 \\
\hline $2012-13$ & 40.91 & 141245 & 33.56 & 115890 & 100 & 345292 \\
\hline $2013-14$ & 38.32 & 137975 & 33.63 & 121059 & 100 & 360025 \\
\hline $2014-15$ & 38.13 & 153709 & 31.75 & 127994 & 100 & 403085 \\
\hline $2015-16$ & 44.59 & 220474 & 26.05 & 128829 & 100 & 494470 \\
\hline $2016-17$ & 52.22 & 293824 & 23.63 & 132933 & 100 & 562639 \\
\hline $2017-18$ & 49.53 & 307423 & 24.14 & 149832 & 100 & 620716 \\
\hline Average & 43.41 & 185137.3 & 30.72 & 121843.6 & 100 & 416131 \\
\hline Source: Handbook & Statistics on Indian Economy, RBI, $2016-17$ & & & \\
\hline & & & & & & \\
\hline
\end{tabular}

Data also shows that the major contributors in the indirect taxes are excise duty and customs duty. After implementing GST its role is shifted to Goods and Service Tax (Brown and Howard, 2002; Sidhartha, 2020) and the share of custom duty moved downwards. In the case of excise duty, its average share has shown an up-down movement. The major reason behind this downward movement was the trade liberalization (Rajan, 2004; Barry, 2008), via, lifting trade barriers in the form of reducing the tariff and quota restrictions.

\section{Trend of non-tax revenue}

Income of the government from sources other than taxes is non-tax revenue. Non-tax revenue become payable only when services offered are availed of, viz; fine, fees, interest receipts, etc. 
Table 6: Trend of non-tax revenue

\begin{tabular}{|c|c|c|c|c|}
\hline \multirow[b]{2}{*}{ Year } & \multicolumn{2}{|c|}{ Interest receipts } & \multicolumn{2}{|c|}{ Non tax revenue } \\
\hline & Percentage & Rs. Crore & Percentage & Rs. Crore \\
\hline $1990-91$ & 72.90 & 8730 & 100 & 11976 \\
\hline $1991-92$ & 68.50 & 10933 & 100 & 15961 \\
\hline $1992-93$ & 62.17 & 12487 & 100 & 20084 \\
\hline $1993-94$ & 68.52 & 15078 & 100 & 22004 \\
\hline $1994-95$ & 66.85 & 15797 & 100 & 23629 \\
\hline $1995-96$ & 65.34 & 18419 & 100 & 28191 \\
\hline $1996-97$ & 67.86 & 22106 & 100 & 32578 \\
\hline $1997-98$ & 66.27 & 25323 & 100 & 38214 \\
\hline 1998-99 & 67.08 & 30076 & 100 & 44833 \\
\hline $1999-00$ & 63.70 & 33895 & 100 & 53211 \\
\hline Average & 66.92 & 19284.4 & 100 & 29068.1 \\
\hline $2000-01$ & 58.65 & 32811 & 100 & 55947 \\
\hline $2001-02$ & 52.44 & 35538 & 100 & 67774 \\
\hline $2002-03$ & 52.04 & 37622 & 100 & 72290 \\
\hline 2003-04 & 50.16 & 38538 & 100 & 76831 \\
\hline $2004-05$ & 39.89 & 32387 & 100 & 81193 \\
\hline $2005-06$ & 28.68 & 22032 & 100 & 76813 \\
\hline $2006-07$ & 27.07 & 22524 & 100 & 83205 \\
\hline $2007-08$ & 20.58 & 21060 & 100 & 102317 \\
\hline 2008-09 & 21.37 & 20717 & 100 & 96940 \\
\hline $2009-10$ & 18.73 & 21784 & 100 & 116275 \\
\hline Average & 36.96 & 28501.3 & 100 & 82958.5 \\
\hline $2010-11$ & 9.03 & 19734 & 100 & 218602 \\
\hline $2011-12$ & 16.64 & 20252 & 100 & 121672 \\
\hline $2012-13$ & 15.11 & 20761 & 100 & 137354 \\
\hline 2013-14 & 11.00 & 21868 & 100 & 198870 \\
\hline 2014-15 & 12.00 & 23734 & 100 & 197766 \\
\hline $2015-16$ & 10.10 & 25378 & 100 & 251260 \\
\hline $2016-17$ & 5.42 & 18149 & 100 & 334770 \\
\hline 2017-18 & 6.59 & 19021 & 100 & 288757 \\
\hline Average & 10.74 & 21112.13 & 100 & 218631.38 \\
\hline
\end{tabular}

In 1990-91 the contribution of non-tax revenue was Rs. 11976 crore and it increased to Rs. 288757 crore in $2017-18$. Up to 2003-04, its contribution to non-tax revenue is greater than 50 percent but after that, it is found decreasing at a higher rate (Table 5).

Public revenue and macro variables in India

The effect of public revenue on selected macroeconomic variables and its effect on public revenue of the central government are attempted, and a two-way analysis is conducted. In the first instance public revenue is chosen as dependent variable and other variables as independent variables. In the second case, macro variable are chosen as dependent variable and public revenue as independent variables, based on the data given in Table 7.

Table 7: Macro economic indicators

\begin{tabular}{|l|c|c|c|c|}
\hline Year & $\begin{array}{c}\text { Public Revenue } \\
\text { (Rs. in Crore) }\end{array}$ & $\begin{array}{c}\text { GDP at Current Price } \\
\text { (Rs. in Crore) }\end{array}$ & $\begin{array}{c}\text { Per Capita Income (or } \\
\text { GNI Per Capita) }\end{array}$ & $\begin{array}{c}\text { Organized Sector } \\
\text { Employment (in Crore) }\end{array}$ \\
\hline $1990-91$ & 93951 & 531813 & 367.56 & 2.674 \\
\hline $1991-92$ & 104558 & 613528 & 303.06 & 2.706 \\
\hline $1992-93$ & 110306 & 703723 & 316.95 & 2.718 \\
\hline $1993-94$ & 130893 & 817961 & 301.16 & 2.738 \\
\hline $1994-95$ & 159778 & 955385 & 346.1 & 2.753 \\
\hline $1995-96$ & 168468 & 1118586 & 373.77 & 2.794 \\
\hline $1996-97$ & 187823 & 1301788 & 399.95 & 2.825 \\
\hline $1997-98$ & 232963 & 1447613 & 415.49 & 2.817 \\
\hline
\end{tabular}




\begin{tabular}{|l|c|c|c|c|}
\hline $1998-99$ & 279549 & 1668739 & 413.3 & 2.811 \\
\hline $1999-00$ & 297189 & 1858205 & 442 & 2.796 \\
\hline $2000-01$ & 326789 & 2000743 & 443.31 & 2.779 \\
\hline $2001-02$ & 363806 & 2175260 & 451.57 & 2.7 \\
\hline $2002-03$ & 411365 & 2343864 & 470.99 & 2.645 \\
\hline $2003-04$ & 475146 & 2625819 & 546.73 & 2.646 \\
\hline $2004-05$ & 506382 & 2971464 & 627.77 & 2.727 \\
\hline $2005-06$ & 526626 & 3390503 & 714.86 & 2.755 \\
\hline $2006-07$ & 578869 & 3953276 & 806.75 & 2.818 \\
\hline $2007-08$ & 739842 & 4582086 & 1028.33 & 2.9 \\
\hline $2008-09$ & 840122 & 5303567 & 998.52 & 2.958 \\
\hline $2009-10$ & 1025874 & 6108903 & 1101.96 & 2.89 \\
\hline $2010-11$ & 1190899 & 7248860 & 1357.56 & 2.9 \\
\hline $2011-12$ & 1320355 & 8391691 & 1458.1 & 2.87 \\
\hline $2012-13$ & 1481383 & 9388876 & 1443.88 & 2.81 \\
\hline $2013-14$ & 1578618 & 10472807 & 1449.61 & 2.86 \\
\hline $2014-15$ & 1585829 & 10527674 & 1573.88 & 1605.61 \\
\hline $2015-16$ & 1790783 & 11369493 & 1732.56 & \\
\hline $2016-17$ & 1975194 & 12308193 & & \\
\hline Source: Computed from Hand book of Statistics on Indian Economy, RBI, 2016-17 & \\
\hline
\end{tabular}

Table 8: Two-way analysis (Model 1)

\begin{tabular}{|c|c|c|c|c|}
\hline \multicolumn{5}{|c|}{ Dependent Variable: $\mathrm{G}=f(\mathrm{PR})$, where, $\mathrm{G}=\mathrm{GDP}, \mathrm{PR}=$ Public Revenue } \\
\hline Variable & Coefficient & Std. Error & t-Statistic & Prob. \\
\hline $\mathrm{C}$ & -83370.46 & 62254.55 & -1.339187 & 0.1926 \\
\hline PR & 6.407462 & 0.069540 & 92.14033 & 0.0000 \\
\hline R-squared & 0.997064 & \multicolumn{2}{|c|}{ Mean dependent var } & 4302979. \\
\hline Adjusted R-squared & 0.996947 & \multicolumn{2}{|c|}{ S.D. dependent var } & 3772361. \\
\hline S.E. of regression & 208454.7 & \multicolumn{2}{|c|}{ Akaike info criterion } & 27.40402 \\
\hline Sum squared resid & $1.09 \mathrm{E}+12$ & \multicolumn{2}{|c|}{ Schwarz criterion } & 27.50001 \\
\hline Log likelihood & -367.9542 & \multicolumn{2}{|c|}{ Hannan-Quinn criter. } & 27.43256 \\
\hline F-statistic & 8489.840 & \multicolumn{2}{|c|}{ Durbin-Watson stat } & 1.074335 \\
\hline Prob(F-statistic) & 0.000000 & & & \\
\hline \multicolumn{5}{|c|}{ Dependent Variable: $\mathrm{P}=f(\mathrm{PR})$, where, $\mathrm{P}=$ Per Capita Income, $\mathrm{PR}=$ Public Revenue } \\
\hline Variable & Coefficient & Std. Error & t-Statistic & Prob. \\
\hline $\mathrm{C}$ & 233.5697 & 24.11518 & 9.685590 & 0.0000 \\
\hline 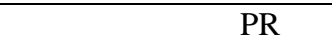 & 0.000822 & $2.69 \mathrm{E}-05$ & 30.49841 & 0.0000 \\
\hline R-squared & 0.973826 & \multicolumn{2}{|c|}{ Mean dependent var } & 795.9758 \\
\hline Adjusted R-squared & 0.972779 & \multicolumn{2}{|c|}{ S.D. dependent var } & 489.4191 \\
\hline S.E. of regression & 80.74787 & \multicolumn{2}{|c|}{ Akaike info criterion } & 11.69173 \\
\hline Sum squared resid & 163005.4 & \multicolumn{2}{|c|}{ Schwarz criterion } & 11.78772 \\
\hline Log likelihood & -155.8383 & \multicolumn{2}{|c|}{ Hannan-Quinn criter. } & 11.72027 \\
\hline F-statistic & 930.1532 & \multicolumn{2}{|c|}{ Durbin-Watson stat } & 0.735268 \\
\hline Prob(F-statistic) & 0.000000 & & & \\
\hline \multicolumn{5}{|c|}{ Dependent Variable: $\mathrm{M}=f(\mathbf{P R})$, where, $\mathrm{M}=$ Employment, $\mathrm{PR}=$ Public Revenue } \\
\hline Variable & Coefficient & Std. Error & t-Statistic & Prob. \\
\hline $\mathrm{C}$ & 2.719999 & 0.019048 & 142.8008 & 0.0000 \\
\hline PR & $9.41 \mathrm{E}-08$ & $2.13 \mathrm{E}-08$ & 4.422482 & 0.0002 \\
\hline R-squared & 0.438938 & \multicolumn{2}{|c|}{ Mean dependent var } & 2.784414 \\
\hline Adjusted R-squared & 0.416495 & \multicolumn{2}{|c|}{ S.D. dependent var } & 0.083494 \\
\hline S.E. of regression & 0.063779 & \multicolumn{2}{|c|}{ Akaike info criterion } & -2.595593 \\
\hline Sum squared resid & 0.101695 & \multicolumn{2}{|c|}{ Schwarz criterion } & -2.499605 \\
\hline Log likelihood & 37.04051 & \multicolumn{2}{|c|}{ Hannan-Quinn criter. } & -2.567051 \\
\hline F-statistic & 19.55835 & \multicolumn{2}{|c|}{ Durbin-Watson stat } & 0.391954 \\
\hline Prob(F-statistic) & 0.000166 & & & \\
\hline
\end{tabular}


Model 2

\begin{tabular}{|c|c|c|c|c|}
\hline \multicolumn{5}{|c|}{ Dependent Variable: $\mathrm{PR}=f(\mathrm{G})$, where, $\mathrm{PR}=$ Public Revenue, $\mathrm{G}=\mathrm{GDP}$} \\
\hline Variable & Coefficient & Std. Error & t-Statistic & Prob. \\
\hline $\mathrm{C}$ & 14983.19 & 9586.169 & 1.563001 & 0.1306 \\
\hline$\overline{\mathrm{G}}$ & 0.155610 & 0.001689 & 92.14033 & 0.0000 \\
\hline R-squared & 0.997064 & \multicolumn{2}{|c|}{ Mean dependent var } & 684568.9 \\
\hline Adjusted R-squared & 0.996947 & \multicolumn{2}{|c|}{ S.D. dependent var } & 587880.1 \\
\hline S.E. of regression & 32485.32 & \multicolumn{2}{|c|}{ Akaike info criterion } & 23.68615 \\
\hline Sum squared resid & $2.64 \mathrm{E}+10$ & \multicolumn{2}{|c|}{ Schwarz criterion } & 23.78214 \\
\hline Log likelihood & -317.7630 & \multicolumn{2}{|c|}{ Hannan-Quinn criter. } & 23.71469 \\
\hline F-statistic & 8489.840 & \multicolumn{2}{|c|}{ Durbin-Watson stat } & 1.077502 \\
\hline Prob(F-statistic) & 0.000000 & & & \\
\hline \multicolumn{5}{|c|}{ Dependent Variable: $\mathrm{PR}=f(\mathrm{P})$, where, $\mathrm{PR}=$ Public Revenue, $\mathrm{P}=\mathrm{Per}$ Capita Income } \\
\hline Variable & Coefficient & Std. Error & t-Statistic & Prob. \\
\hline $\mathrm{C}$ & -258945.3 & 36131.65 & -7.166718 & 0.0000 \\
\hline $\mathrm{P}$ & 1185.355 & 38.86613 & 30.49841 & 0.0000 \\
\hline R-squared & 0.973826 & \multicolumn{2}{|c|}{ Mean dependent var } & 684568.9 \\
\hline Adjusted R-squared & 0.972779 & \multicolumn{2}{|c|}{ S.D. dependent var } & 587880.1 \\
\hline S.E. of regression & 96992.67 & \multicolumn{2}{|c|}{ Akaike info criterion } & 25.87385 \\
\hline Sum squared resid & $2.35 \mathrm{E}+11$ & \multicolumn{2}{|c|}{ Schwarz criterion } & 25.96983 \\
\hline Log likelihood & -347.2969 & \multicolumn{2}{|c|}{ Hannan-Quinn criter. } & 25.90239 \\
\hline F-statistic & 930.1532 & \multicolumn{2}{|c|}{ Durbin-Watson stat } & 0.729316 \\
\hline Prob(F-statistic) & 0.000000 & & & \\
\hline \multicolumn{5}{|c|}{ Dependent Variable: $\mathrm{PR}=f(\mathrm{M})$, where, $\mathrm{PR}=$ Public Revenue, $\mathrm{M}=$ Employment } \\
\hline Variable & Coefficient & Std. Error & t-Statistic & Prob. \\
\hline $\mathrm{C}$ & -12304183 & 2938254. & -4.187584 & 0.0003 \\
\hline $\mathrm{M}$ & 4664806. & 1054794. & 4.422482 & 0.0002 \\
\hline R-squared & 0.438938 & \multicolumn{2}{|c|}{ Mean dependent var } & 684568.9 \\
\hline Adjusted R-squared & 0.416495 & \multicolumn{2}{|c|}{ S.D. dependent var } & 587880.1 \\
\hline S.E. of regression & 449066.7 & \multicolumn{2}{|c|}{ Akaike info criterion } & 28.93892 \\
\hline Sum squared resid & $5.04 \mathrm{E}+12$ & \multicolumn{2}{|c|}{ Schwarz criterion } & 29.03491 \\
\hline Log likelihood & -388.6754 & \multicolumn{2}{|c|}{ Hannan-Quinn criter. } & 28.96746 \\
\hline F-statistic & 19.55835 & \multirow{2}{*}{\multicolumn{2}{|c|}{ Durbin-Watson stat }} & 0.194186 \\
\hline Prob(F-statistic) & 0.000166 & & & \\
\hline
\end{tabular}

Model 3

\begin{tabular}{|c|c|c|c|c|}
\hline \multicolumn{5}{|c|}{ Dependent Variable: $P R=f(G, P, M)$} \\
\hline \multicolumn{5}{|c|}{ Method: Least Squares } \\
\hline \multicolumn{5}{|c|}{ Included observations: 27} \\
\hline Variable & Coefficient & Std. Error & t-Statistic & Prob. \\
\hline $\mathrm{C}$ & 252976.9 & 303336.1 & 0.833982 & 0.4133 \\
\hline G & 0.129582 & 0.029748 & 4.356002 & 0.0003 \\
\hline $\mathrm{P}$ & 119.3622 & 85.60578 & 1.394324 & 0.1771 \\
\hline $\mathrm{M}$ & -97635.11 & 112324.8 & -0.869222 & 0.3941 \\
\hline R-squared & 0.997379 & \multicolumn{2}{|c|}{ Mean dependent var } & 684568.9 \\
\hline Adjusted R-squared & 0.996903 & \multicolumn{2}{|c|}{ S.D. dependent var } & 587880.1 \\
\hline S.E. of regression & 32717.53 & \multicolumn{2}{|c|}{ Akaike info criterion } & 23.79479 \\
\hline Sum squared resid & $2.35 \mathrm{E}+10$ & \multicolumn{2}{|c|}{ Schwarz criterion } & 24.03476 \\
\hline Log likelihood & -316.2296 & \multicolumn{2}{|c|}{ Hannan-Quinn criter. } & 23.86614 \\
\hline F-statistic & 2093.101 & \multicolumn{2}{|c|}{ Durbin-Watson stat } & 1.089759 \\
\hline Prob(F-statistic) & 0.000000 & & & \\
\hline
\end{tabular}

Tax buoyancy is an indicator to measure efficiency to growth in GDP. If tax buoyancy is high, it indicates more than proportionate response of tax revenue to rise in GDP. It is computed by dividing the percentages in tax revenue by the percentage changes in GDP over period (Sonika Gupta K. S, 2016). Gross tax buoyancy coefficient remained fluctuating during the period 1990-91 to 2016-17. It was 1.1 in 1991-92 indicate positive response to growth in GDP. Then it increased 
to 2.45 in 2002-03, again reduced to 0.07 in 2008-09. Obviously, the highest buoyancy coefficient of 2.45 was in $2002-03$ and lowest coefficient in 2001-02 with a value of -0.27 , indicating negative response to growth in GDP (Table 9). The figures also reveal that the direct tax is showing more response to GDP than indirect tax.

Table 9: Buoyancy coefficient of Major taxes of Central government (with respect to GDP)

\begin{tabular}{|l|c|c|c|}
\hline \multirow{2}{*}{ Year } & \multicolumn{3}{|c|}{ Buoyancy Coefficient } \\
\hline $1990-91$ & Direct Tax & Indirect Tax & Gross Tax \\
\hline $1991-92$ & - & - & - \\
\hline $1992-93$ & 3.10 & 0.72 & 1.10 \\
\hline $1993-94$ & 1.31 & 0.34 & 0.53 \\
\hline $1994-95$ & 0.25 & -0.16 & -0.07 \\
\hline $1995-96$ & 2.72 & 1.15 & 1.51 \\
\hline $1996-97$ & 1.22 & 1.25 & 1.24 \\
\hline $1997-98$ & 0.88 & 0.93 & 0.91 \\
\hline $1998-99$ & 0.66 & 0.02 & 0.20 \\
\hline $1999-00$ & 1.24 & 0.40 & 0.64 \\
\hline $2000-01$ & 2.38 & 1.62 & 1.85 \\
\hline $2001-02$ & 2.69 & 0.03 & 0.89 \\
\hline $2002-03$ & -0.46 & -0.16 & -0.27 \\
\hline $2003-04$ & 3.81 & 1.69 & 2.45 \\
\hline $2004-05$ & 2.02 & 1.15 & 1.49 \\
\hline $2005-06$ & 1.79 & 1.19 & 1.43 \\
\hline $2006-07$ & 1.85 & 1.16 & 1.45 \\
\hline $2007-08$ & 2.50 & 1.31 & 1.84 \\
\hline $2008-09$ & 2.26 & 0.91 & 1.56 \\
\hline $2009-10$ & 0.56 & -0.48 & 0.07 \\
\hline $2010-11$ & 0.63 & -0.35 & 0.20 \\
\hline $2011-12$ & 0.76 & 1.92 & 1.23 \\
\hline $2012-13$ & 0.60 & 0.75 & 0.67 \\
\hline $2013-14$ & 1.50 & 1.98 & 1.72 \\
\hline $2014-15$ & 1.15 & 0.33 & 0.77 \\
\hline $2015-16$ & 0.91 & 1.11 & 1.00 \\
\hline $2016-17$ & -1.03 & 1.26 & 0.45 \\
\hline Source: Computed on the basis & 1.56 & & 1.40 \\
\hline & & & \\
\hline
\end{tabular}

\section{Conclusion}

Total public revenue of the central government had been continuously increasing since 1990, especially the revenue receipts. There was a rapid increase in the total receipt from Rs. 93951 crore in $1990-91$ to 2315113 crore in 2018-19, registering 24 times growth. Similarly, the share of tax revenue increased from Rs. 42978 crore in 1990 to Rs. 918423 crore in 2018-19. There is a rapid increase in the share of direct taxes in total tax revenue compared to indirect taxes. This is due to the increasing share of personal income tax. The corporation tax contributed a higher share in total direct taxes with 74.98 percent; 62.21 percent and 61 percent in the first, second and third phase, respectively. The share of indirect taxes also shows a rapid increase in the total tax revenue from Rs. 36075 crore in 1990-91 to Rs. 620716 crore in $2017-18$. In the first phase the average contribution on indirect taxes to total tax revenue was Rs. 56382 crore, which is greater than the average share of direct taxes in the same phase and this trend continued in the consecutive two time phases also. After implementing GST its role is shifted to Goods and Service Tax. The capital receipt shows an increase from time to time. The average amount of capital receipt in the first phase was Rs. 70256 crore and it increased to 261387 crore in second and it again increased to Rs. 507727 crore in third phase but compared to revenue receipt, the share of capital receipt decreased over time. The two-way regression analysis reveals that Public Revenue and macroeconomic indicators are significantly interlinked and correlated. Gross tax buoyancy coefficient remained fluctuating during the period 1990-91 to 2016-17. Obviously, the highest buoyancy coefficient of 2.45 was in 2002-03 and lowest in 2001-02 with a value of 0.27 , indicating negative response to growth in GDP, revealed direct tax more response to GDP than indirect tax.

\section{Source of Funding}

None.

\section{Conflict of Interest}

The authors declare no conflict of interest. 


\section{References}

1. Barry Bosworth, S. M. Accounting for Growth: Comparing China and India. J Econ Perspect. 2008;22:45-66.

2. Brown JL, Howard LR. (2002), Principles and Practice of Management Accountancy. Macdonald and Evans Ltd, London.

3. Devasia MD, Karunakaran N, Prathap MV. Public Expenditure of India: Trend and Effects. J Manag Res Anal. 2020;7(3):11421.

4. Odedokun MO. (2001) Public Finance and Economic Growth, Discussion Paper", World Institute for Development Economic Research.

5. Goyal R, Khundrakpam J, Ray P. Is India's Public Finance Unsustainable? Or, are the Claims Exaggerated. J Policy Modeling. 2004;26(3)401-420.
6. Seligman ER. Bastable's Public Finance. Political Science Quarterly. 1892;708-720

7. Sidhartha (2020), Why Number of Income Tax Payers Halved in Just one Year in India.

8. Gupta S, Singh K. Fiscal Deficit and its Trend in India. Int J Business Manag Invention. 2016;5(11):63-75.

How to cite this article: Devasia MD, Karunakaran N. Public revenue in India: Trend and effect. J Manag Res Anal. 2020;7(4):172-82. 